\title{
Development and Validation of a Clinical-Radiomic Model for Preoperative Prediction of Lymph Node Metastasis and Overall Survival in Rectal Cancer: A Retrospective Study
}

\section{Yuzhou Zhu}

Department of Gastrointestinal Surgery, West China Hospital, Sichuan University, No. 37 Guo Xue Alley, Chengdu 610041, Sichuan Province, China. West China Medical School, Sichuan University, Chengdu, Sichuan Province, China

\section{Kaibo Sun}

West China Medical School, Sichuan University, Chengdu, Sichuan Province, China

\section{Tinghan Yang}

Department of Gastrointestinal Surgery, West China Hospital, Sichuan University, No. 37 Guo Xue Alley, Chengdu 610041, Sichuan Province, China. West China Medical School, Sichuan University, Chengdu, Sichuan Province, China

\section{Qingbin Wu}

Department of Gastrointestinal Surgery, West China Hospital, Sichuan University, No. 37 Guo Xue Alley, Chengdu 610041, Sichuan Province, China. West China Medical School, Sichuan University, Chengdu, Sichuan Province, China

\section{Yinzhi Shen}

West China Medical School, Sichuan University, Chengdu, Sichuan Province, China

\section{Zixin Zhang}

West China Medical School, Sichuan University, Chengdu, Sichuan Province, China

\section{Xubing Zhang}

West China Medical School, Sichuan University, Chengdu, Sichuan Province, China

\section{Ziqiang Wang ( $\nabla$ wangziqiang@scu.edu.cn )}

Sichuan University West China Hospital https://orcid.org/0000-0002-2874-1535

\section{Research}

Keywords: rectal cancer, clinical-radiomic model, lymph node metastasis, prognosis

Posted Date: June 30th, 2020

DOI: https://doi.org/10.21203/rs.3.rs-37253/v1 
License: (c) (i) This work is licensed under a Creative Commons Attribution 4.0 International License. Read Full License 


\section{Abstract}

Background: Preoperative lymph node (LN) metastasis is essential to therapeutic strategies for rectal cancer (RC) patients without distal metastasis, and is one of the major predictive risk factors. Unfortunately, it is difficult to predict the preoperative LN metastasis. Thus, we established a clinicalradiomic model to assist in predicting LN metastasis and overall survival (OS) in RC patients preoperatively.

Methods: The prediction model was established and validated in a primary cohort consisting of 150 patients with pathologically confirmed RC, and data was gathered from January 2013 and December 2015. Radiomic features were extracted from the CT images of portal venous phase (PVP). For selection of the features, LASSO regression method was used. We established a clinical-radiomic model by incorporating the radiomic features and independent clinical risk factors. Then we adopted this combined model to classify RC patients into high- and low-risk groups for 3-year OS analysis.

Results: A total of 49 patients were proved to have LN metastasis pathologically, and 101 patients had no metastasis of LN. The Rad-score consisted of 2 selected features. The combined clinical-radiomic model included preoperative clinical T stage, preoperative clinical N stage and the Rad-score. The combined model showed good performance of discrimination, with a AUC of 0.847 in the training cohort and 0.782 in the validation cohort. Significant differences existed in OS between high- and low-risk groups in both the training $(p=0.026)$ and validation $(p=0.034)$ cohort.

Conclusion: This combined clinical-radiomic model could be conveniently used to facilitate the preoperative prediction of $\mathrm{LN}$ metastasis and prognosis in patients with RC.

Trial registration: This research was retrospectively registered at our institution's medical ethics committee (NO.2019-1159)

\section{Background}

Rectal cancer (RC) is the third leading cause of cancer-related death worldwide In. The incidence of RC is increasing, particularly in younger age groups (1). Moreover, local recurrence or metastatic disease can occur in up to $30 \%$ of patients within five years of surgery $(2,3)$.

Lymph node (LN) metastasis is crucial for determining the therapeutic strategy and constitutes one of the major prognostic risk factors in patients having RC without distal metastasis $(4,5)$. Magnetic resonance imaging (MRI) and Multiple-detector computed tomography (MDCT) are major methods for pretreatment assessment. Unfortunately, there is no consensus standard to predict metastatic LNs (6-8). The maximum short-axis diameters of LNs were commonly used to identify malignant LNs in previous studies $(6,9)$. However, there is significant size overlap between benign and malignant LNs (10), and over 50 percent of the LNs involved in RC are less than $5 \mathrm{~mm}$ (11). 
Consequently, better tools for preoperative prediction of LN metastasis is still in need. Moreover, prognosis of RC patients after operation varies, making better risk stratification methods important.

Radiomics, which involves texture analysis, is a high-throughput approach allowing clinicians to extract large amounts of quantitative data from images, thus providing information that cannot be visually assessed but could be related to the tumor phenotype $(12,13)$. The prognostic performance of radiomics based on $\mathrm{CT}$ images, has been evidenced in several cancers, including pancreatic cancer, lung cancer and esophageal cancer (14-16). Therefore, we aimed to develop and validate a combined clinical-radiomic model to preoperatively predict lymph node status for patients having RC without distal metastasis, and to associate the combined model with overall survival (OS).

\section{Method}

This research was approved by our institution's medical ethics committee (NO.2019-1159). The need for informed consent was waved.

\section{Patients}

By searching electronic medical records, patients with histologically confirmed RC who underwent resection in our institution were recruited. Inclusion criteria: (1) patients were treated with resection; (2) preoperative enhancement CT was conducted within 1 month prior to surgical resection; (3) follow-up was performed for at least 3 years; (4) LN metastasis was confirmed by pathology. Exclusion criteria: (1) lack of preoperative CT images; (2) patients with distal metastasis; (3) loss of follow-up; (4) poor CT image quality. Patients were assigned to a training and a validation cohort at a ratio of 7:3 by the date of scanning. The flow-diagram of patient inclusion process was presented in Figure 1.

\section{CT Examination and Imaging Analysis}

Although the design of this study was retrospective, only one CT scanner was chosen: 128-MDCT scanner (Somatom Definition AS+; Siemens Healthcare Sector, Forchheim, Germany). The scanning parameters: amperage, 200-210 mA; voltage, $120 \mathrm{kV}$; pitch, 1.0; rotation time, 0.5 seconds; and section thickness, $2.0 \mathrm{~mm}$. Patients were injected an anionic contrast medium (Omnipaque 350; GE Healthcare, Chicago, IL) intravenously, at a dose of $1.5 \mathrm{~mL} / \mathrm{kg}$, and at a rate of $3 \mathrm{~mL} / \mathrm{s}$. With the trigger threshold of the aorta reaching $100 \mathrm{HU}$, a three-phase scan was obtained. However, only the PVP image was used to extract texture parameters.

\section{Follow-up}

The end point of this study was OS, which was defined as the time from the date of RC resection until patients died, or until the day the patient was last known to be relapse-free. All patients were followed up 
continuously for at least 3 years, until the patient died or lost. Postoperative recurrence was monitored by standard NCCN guidelines, including clinical evaluation, CEA, CA19-9 every 3 months for the first year and every 6 months until the $3^{\text {rd }}$ year (17). Other standard testing included yearly CT scans as well as surveillance colonoscopy until the $3^{\text {rd }}$ year(17).

\section{Radiomic Features Extraction}

By using ITK-Snap software (available at www.itk-snap.org) \a radiologist manually delineated the regions of interest (ROIs) within a tumor (about $1 \mathrm{~mm}$ from the tumor margin)(Figure 2) to recognize the three-dimensional (3D) volume area in PVP images(18). Calcification, blood vessels and cystic areas within the tumor were excluded. Texture characteristic were extracted and analyzed from all patients by using A.K. software (Analysis-Kit; GE Healthcare). 396 texture features of six kinds were extracted: Gray level histogram, Haralick features, gray level co-occurrence matrix (GLCM), gray level size zone matrix, run-length matrix, and form factor feature. The histogram showed the intensity of the pixels and the runlength matrix in particular directions(19). The Haralick features and co-occurrence matrix presented information on the distribution of the gray level value in all directions of the pixel pairs(20). The gray level size zone matrix was effective for characterizing texture homogeneity, or a speckle-like texture $(32,33)$. By using the histogram features, we extracted the parameters of radiomic features and produced a qualitative or quantitative description. A series of matrix transformations such as gray level cooccurrence metrics and run-length metrics reflected the transform matrix texture of high-level ROI. We analyzed the ROI characteristics across various resolution levels with the wavelet transformation. With the filter-transform texture, we obtained a set of target features by using various filter types such as log transformation and Gaussian transformation(21).

\section{Model-building and evaluation}

Because of the large number of texture features and the relatively limited size of the cohort, redundant texture features need to be eliminated. Therefore, we analyzed all radiomic characteristics for the correlation of any two features, and features were redundant if the coefficient of linear correlation was above 0.6(22). In order to avoid overfitting the high-dynamic data analysis, we adopted the least absolute regression shrinkage and selection operators (LASSO)(22). The optimization of the tuning parameter $(\lambda)$ in the LASSO regression reduced the majority of the characteristics to zero and selected the remaining features with non-zero coefficients.(22) The most distinctive features were therefore identified. The stability of these features was assessed by calculating the intraclass correlation coefficient (ICC) from 30 patients(23). This features with ICC $>0.75$ were included. Each radiomic feature was evaluated by univariate logistic regression. To avoid missing important features, statistical importance was assumed at 0.2(24). The features which were statistically significant with univariate logistic regression analysis, were then evaluated for model building with multivariate logistic regression analysis. Then, radiomics signature was created with a radiomics score (Rad-score). And a combined model was created to predict 
LN status with incorporation of the preoperative clinical risk factors and Rad-score. Eventually, we established a nomogram for model visualization, and performed the receiver operating characteristic (ROC) curve analysis to evaluated the diagnostic performance to predict LN metastasis of RC. We also conducted Kaplan-Meier (K-M) survival curves to investigate the association of the predicting models with 3-year OS of RC patients.

\section{Statistical analysis}

All data analyzes were performed using SPSS version 22 (SPSS Inc., Chicago, IL), R software version 3.3.2 (R Foundation for Statistical Computing, Vienna, Austria), and Medcalc v.15.8 (Medcalc Software, Ostend, Belgium). Mann-Whitney $U$ test and $X^{2}$ test were used to assess if the values of preoperative clinical variables were significantly different between training and validation group. Two-sided $p$ value less than 0.05 was considered as significantly different.

\section{Results}

A total of 49 patients were proved to have $L N$ metastasis pathologically, and 101 patients had no metastasis of LN. The baseline information of each included patients, including preoperative clinical T stage (cT stage), preoperative clinical $\mathrm{N}$ stage (cN stage), preoperative overall stage, age, sex and followup time were shown in table 1. 


\begin{tabular}{|c|c|c|c|}
\hline Characteristics & Training $(n=105)$ & Validation $(n=45)$ & $p$ Value \\
\hline Age (y) & $59 \pm 12$ & $61 \pm 11$ & 0.242 \\
\hline Gender & & & 0.232 \\
\hline Male & 50 & 28 & \\
\hline Female & 55 & 17 & \\
\hline Clinical T stage & & & 0.445 \\
\hline $\mathrm{T} 1$ & 14 & 1 & \\
\hline T2 & 61 & 31 & \\
\hline T3 & 25 & 13 & \\
\hline T4 & 5 & 0 & \\
\hline Clinical N stage & & & 0.990 \\
\hline No & 69 & 29 & \\
\hline N1 & 30 & 15 & \\
\hline N2 & 6 & 1 & \\
\hline Clinical M stage & 0 & 0 & \\
\hline Pathological N stage* & & & 0.909 \\
\hline LN metastasis (N1/N2) & $34(22 / 12)$ & $15(8 / 7)$ & \\
\hline Non-metastasis (N0) & 71 & 30 & \\
\hline Overall Stage (cTNM) & & & 0.451 \\
\hline$\square$ & 57 & 20 & \\
\hline$\square$ & 12 & 9 & \\
\hline$\square$ & 36 & 16 & \\
\hline$\square$ & 0 & 0 & \\
\hline \multicolumn{4}{|l|}{ Follow-up time (months) } \\
\hline OS & $33.8 \pm 5.4$ & $34.0 \pm 5.2$ & 0.774 \\
\hline
\end{tabular}




\section{Feature Selection and Model-building}

After deleting the redundant features, a total of 7 radiomic features were remained. The feature "Compactness2" was selected by LASSO method from the remaining features (Figure 3). To avoid missing important features, another one feature "ShortRunEmphasis_AllDirection_offset4_SD" was selected by univariate logistic regression $(P=0.057<0.2)$. These two features were presented in this calculation formula: Rad-score $=1 /(1+\operatorname{EXP}(-(-0.798-$

$0.445 \times$ Compactness $2+0.332 \times$ ShortRunEmphasis_AllDirection_offset4_SD $))$ ). The radiomics signature (Rad-score) was statistically different between LN metastasis and non-metastasis groups (Rad-score = $0.37 \pm 0.14$, and $0.30 \pm 0.12$ respectively; $P=0.003$ ).

As for clinical features, preoperative $\mathrm{cT}$ stage, and preoperative $\mathrm{cN}$ stage were selected by univariate logistic regression analysis $(P=0.002, P=0.000$, respectively). Thus the combined clinical-radiomic model was built by multivariate logistic regression analysis, which was shown in this formula: $1 /(1+\operatorname{EXP}(-$ $(-5.191+0.703 \times c$ T stage $+2.257 \times \mathrm{cN}$ stage $+5.425 \times$ Rad-score $)))$. The results of this combined model between LN metastasis and non-metastasis groups were significantly different $(0.55 \pm 0.32,0.21 \pm 0.18, \mathrm{P}<$ 0.001). The Hosmer-Lemeshow test indicated no statistical significance $(P=0.453,0.416$ for Rad-score and the combined model, respectively), which suggested good fit of these models(25).

A nomogram was established for the combined model visualization (Figure 4). To use the nomogram, the values on the respective axis for each variables need to be found and added together. Then by drawing a line from the total points axis to the axis of probability, the risk for $L N$ metastasis can be assessed. Higher overall score was correlated with higher risk of LN metastasis. The contributions of these factors to the Rad-score and the combined model were measured by the standardized logistic regression coefficient. The contribution of "Compactness2" to the Rad-score was greater than another one (Figure 5), and that of "cN stage" to the combined model was highest (Figure 5).

\section{Model for LN status prediction}

If only radiomics signature (Rad-score) was considered, the AUC was 0.674 ( $95 \% \mathrm{Cl}, 0.575-0.762$ ) (Figure $6 \&$ Table 2). In order to improve predictive performance for $L N$ status, the radiomic features could be combined with preoperative clinical characteristics. The AUCs of the combined model and the clinical model (preoperative clinical T stage and $\mathrm{N}$ stage) were $0.847(95 \% \mathrm{Cl}, 0.764-0.910)$ and $0.818(95 \% \mathrm{Cl}$, 0.731-0.887) (Figure $6 \&$ Table 2), respectively. The clinical variables contributed better to the combined model than the Rad-score, which corresponded to the higher standardized logistic regression coefficient of preoperative $\mathrm{cN}$ stage than that of Rad-score (Figure 5). Although clinical model showed higher AUC value than Rad-score, the Rad-score helped greatly to increase the AUC value from 0.818 to 0.847 . 
Table 2. The diagnostic performance of the three models in both training and validation cohorts

\begin{tabular}{|lllllll|}
\hline model & radscore & clinical & combined & radscore & clinical & combined \\
\hline AUC & training set & & \multicolumn{5}{c|}{ validation set } \\
SEN & 9.674 & 0.818 & 0.847 & 0.698 & 0.739 & 0.782 \\
\hline SPE & $35.21 \%$ & $85.92 \%$ & $87.32 \%$ & $86.67 \%$ & $56.67 \%$ & $83.33 \%$ \\
\hline $\begin{array}{l}\text { AUC = area under the summary receiver operating characteristic curve; SEN = sensitivity; SPE = } \\
\text { specificity }\end{array}$ & & $70.59 \%$ & $73.53 \%$ & $53.33 \%$ & $80 \%$ & $66.67 \%$ \\
\hline
\end{tabular}

\section{Combined model performance for OS}

The combined model stratified patients into low-risk and high-risk groups. Significant differences existed in OS of predicted low-risk and high-risk groups in both the training $(p=0.026)$ and validation $(p=0.034)$ cohort (Figure 7).

\section{Discussion}

Preoperative LN stage is essential to therapeutic strategy, and is one of the major predictive risk factors for RC patients without distal metastasis. Unfortunately, it was difficult to predict the LN metastasis through all existing methods (26), thus we constructed a combined clinical-radiologic model to preoperatively predict LN status, and to classify patients into high- and low-risk classes for 3-year OS analysis. Our results suggested that the combined model could improve diagnostic performance compared to the model with clinical risk factors alone, and 3-year OS between high- and low-risk groups were significantly different in both training and validation cohort.

Compared with traditional imaging methods to assess anatomical changes of $L N$, the advantage of radiomics is using large numbers of quantitative features to reflect the inherent heterogeneity of the lesion $(27,28)$, that is invisible to human eyes. Recently, radiomic analysis has emerged as a powerful method for developing decision-making models. Radiomic-based predictive models for advanced nasopharyngeal carcinoma, early-stage non-small cell lung cancer, and rectal cancer (14-16) have been reported. Our study demonstrated that 2 robust radiomic features were associated with LN status and prognosis for RC patients.

Regarding the clinical features, preoperative $\mathrm{cT}$ and $\mathrm{cN}$ stage had high standardized logistic regression coefficient to the combined model, which was in line with the common sense that cT and cN stage had a significant correlation with pathological LN metastasis. Other clinical risk factors were not associated with LN metastasis and 3-year OS significantly. 
In this study, the AUC value of rad-score was lower than that of clinical risk factors. This might be because we included RC patients at various stage, and the disparity in clinical features was greater than that in radiomic features among $\mathrm{RC}$ at various stages. Even so, the contribution of rad-score to the combined model was greater than that of cT stage. Moreover, the combination of rad-score helped to improve the AUC value from 0.818 to 0.847 in the training cohort and from 0.739 to 0.782 in the validation cohort.

Compared with some previous articles focused on the similar topic $(21,24)$, our combined model showed better predictive value. The reason for this is may be the better data processing flow and control of heterogeneity. First, LASSO method not only succeeds the method of selecting predictors based on the strength of their univariable association with outcome, but also allows the combination of the panel of selected characteristics into a radiomic signature. Furthermore, all CT images used in our research were

obtained from the same CT scanner, and patients were treated only with surgery. Besides better predictive value, our research associated the combined model with prognosis.

However, there were several limitations in this study. Firstly, as a retrospective study, there could be unavoidable selection bias, therefore, prospective and external validation studies are necessary. Secondly, this research resulted from a single institution, so multicenter validation is needed to expand the flexibility of the experimental results. Thirdly, this study utilized only one imaging modality, which contributes to a limited number of extracted radiomics features. If more imaging modalities (such as MRI and PET-CT) are integrated, the feature pool could be extended effectively to provide more useful radiomics information.

\section{Conclusions}

This clinical-radiomic model could be conveniently used to promote the preoperative prediction of LN metastasis and prognosis in RC patients.

\section{List Of Abbreviations}

$\mathrm{LN}=$ lymph node

$\mathrm{RC}=$ rectal cancer

OS = overall survival

$\mathrm{PVP}=$ portal venous phase

MRI = Magnetic resonance imaging

MDCT = Multiple-detector computed tomography

$\mathrm{ROI}=$ regions of interest 
LASSO = least absolute regression shrinkage and selection operators

ICC = intraclass correlation coefficient

$\mathrm{ROC}=$ receiver operating characteristic

\section{Declarations}

\section{Ethics approval and consent to participate}

This research was approved by our institution's medical ethics committee (NO.2019-1159). The need for informed consent was waved.

\section{Consent for publication}

Not applicable

\section{Availability of data and materials}

The datasets used and/or analysed during the current study are available from the corresponding author at wangziqiang@scu.edu.cn on reasonable request.

\section{Competing interests}

The authors declare that they have no competing interests

\section{Funding}

This work was supported by 1·3.5 Project for Disciplines of Excellence - Clinical Research Incubation Project, West China Hospital, Sichuan University; under Grant No. 2019HXFH03; and Ministry of Science and Technology of the People's Republic of China; under Grant No. 2017 YFC0908204.

\section{Contributions}

YZ designed the study and wrote the manuscript. KS drew the region of interest and revised the manuscript. TY and QW reviewed the ROI drawn by KS. YS and ZZ analyzed the radiomic data. XZ analyzed the clinical data. ZW reviewed and approved the final manuscript. All authors read and approved the final manuscript. 


\section{Acknowledgements}

Not applicable

\section{References}

1. EM S, CC M. Epidemiology and Mechanisms of the Increasing Incidence of Colon and Rectal Cancers in Young Adults. Gastroenterology. 2020;158(2):341-53.

2. PA K, M T, P N, J S, EM G, V A, et al. Health-Related Quality of Life in MONARCH 2: Abemaciclib plus Fulvestrant in Hormone Receptor-Positive, HER2-Negative Advanced Breast Cancer After Endocrine Therapy. The oncologist. 2019; undefined(undefined):undefined.

3. F D, TR C, SA A, NN B. A watch-and-wait approach for locally advanced rectal cancer after a clinical complete response following neoadjuvant chemoradiation: a systematic review and meta-analysis. The lancet Gastroenterology \& hepatology. 2017;2(7):501-13.

4. AJ S, DK D, K S, A H, RS M, M S, et al. Guideline for optimization of colorectal cancer surgery and pathology. Journal of surgical oncology. 2010;101(1):5-12.

5. Y T, Y I, T S, H F, S S, J H, et al. Serum Angiopoietin-like Protein 2 Improves Preoperative Detection of Lymph Node Metastasis in Colorectal Cancer. Anticancer research. 2015;35(5):2849-56.

6. XT L, YS S, L T, K C, XY Z. Evaluating local lymph node metastasis with magnetic resonance imaging, endoluminal ultrasound and computed tomography in rectal cancer: a meta-analysis. Colorectal disease : the official journal of the Association of Coloproctology of Great Britain and Ireland. 2015;17(6):0129-35.

7. J C, SN O, DM Y, WK K, CK J, SW K, et al. Computed tomography and magnetic resonance imaging evaluation of lymph node metastasis in early colorectal cancer. World journal of gastroenterology. 2015;21(2):556-62.

8. TJ V, W P, MG M, M H, R H, A A, et al. Accuracy of staging rectal tumors with contrast-enhanced transrectal MR imaging. AJR American journal of roentgenology. 1997;168(6):1427-34.

9. S O, J H, H I, T K, M O, E S, et al. The important risk factor for lateral pelvic lymph node metastasis of lower rectal cancer is node-positive status on magnetic resonance imaging: study of the Lymph Node Committee of Japanese Society for Cancer of the Colon and Rectum. International journal of colorectal disease. 2016;31(10):1719-28.

10. J H, AC B, NJ C, I R. Lymph node revealing solutions in colorectal cancer: should they be used routinely? Journal of clinical pathology. 2014;67(5):383-8.

11. G L, A P, DM B. Size and distribution of lymph nodes in rectal cancer resection specimens. Diseases of the colon and rectum. 2015;58(4):406-14.

12. M H, F T, D V, C CLR. Radiomics in PET/CT: More Than Meets the Eye? Journal of nuclear medicine : official publication, Society of Nuclear Medicine. 2017;58(3):365-6. 
13. $P$ L, E R-V, R L, S C, RG vS, P G, et al. Radiomics: extracting more information from medical images using advanced feature analysis. European journal of cancer (Oxford, England : 1990). 2012;48(4):441-6.

14. Z Y, J Z, H L, X S, D L, T T, et al. Correlation of CT texture changes with treatment response during radiation therapy for esophageal cancer: An exploratory study. PloS one. 2019;14(9):e0223140.

15. MMK K, L N, IR V, FL A, G C, V G, et al. Heterogeneity in tumours: Validating the use of radiomic features on F-FDG PET/CT scans of lung cancer patients as a prognostic tool. Radiotherapy and oncology : journal of the European Society for Therapeutic Radiology and Oncology. 2020;144:72-8.

16. HS K, YJ K, KG K, JS P. Preoperative CT texture features predict prognosis after curative resection in pancreatic cancer. Scientific reports. 2019;9(1):17389.

17. AB B, AP V, MM A-H, L C, YJ C, KK C, et al. Rectal Cancer, Version 2.2018, NCCN Clinical Practice Guidelines in Oncology. Journal of the National Comprehensive Cancer Network : JNCCN. 2018;16(7):874-901.

18. PA Y, J P, HC H, RG S, S H, JC G, et al. User-guided 3D active contour segmentation of anatomical structures: significantly improved efficiency and reliability. Neurolmage. 2006;31(3):1116-28.

19. KA M, B G, MP H. CT texture analysis using the filtration-histogram method: what do the measurements mean? Cancer imaging : the official publication of the International Cancer Imaging Society. 2013;13(3):400-6.

20. T H, MD M, N S, TA F, L L, RE T. Can Quantitative CT Texture Analysis be Used to Differentiate Fatpoor Renal Angiomyolipoma from Renal Cell Carcinoma on Unenhanced CT Images? Radiology. 2015;276(3):787-96.

21. Li M, Zhang J, Dan Y, Yao Y, Dai W, Cai G, et al. A clinical-radiomics nomogram for the preoperative prediction of lymph node metastasis in colorectal cancer. J Transl Med. 2020;18(1):46.

22. P T, VM T. Parsimonious and robust multivariate calibration with rational function Least Absolute Shrinkage and Selection Operator and rational function Elastic Net. Analytica chimica acta. 2013;768:57-68.

23. J B, WE dB, JW B, JL H, S K, R C, et al. Inter-rater agreement in evaluation of disability: systematic review of reproducibility studies. BMJ (Clinical research ed). 2017;356:j14.

24. Huang YQ, Liang CH, He L, Tian J, Liang CS, Chen X, et al. Development and Validation of a Radiomics Nomogram for Preoperative Prediction of Lymph Node Metastasis in Colorectal Cancer. J Clin Oncol. 2016;34(18):2157-64.

25. AA K, JE Z. Assessing the calibration of mortality benchmarks in critical care: The HosmerLemeshow test revisited. Critical care medicine. 2007;35(9):2052-6.

26. RJ G, PE K, H H. Radiomics: Images Are More than Pictures, They Are Data. Radiology. 2016;278(2):563-77.

27. C S, H W, M A-N, G P. Preoperative CT-based predictive factors for resectability and medium-term overall survival in patients with peritoneal carcinomatosis from colorectal cancer. Clinical radiology. 2018;73(8):756.e11-.e16. 
28. Z L, XY Z, YJ S, L W, HT Z, Z T, et al. Radiomics Analysis for Evaluation of Pathological Complete Response to Neoadjuvant Chemoradiotherapy in Locally Advanced Rectal Cancer. Clinical cancer research : an official journal of the American Association for Cancer Research. 2017;23(23):7253-62.

\section{Figures}

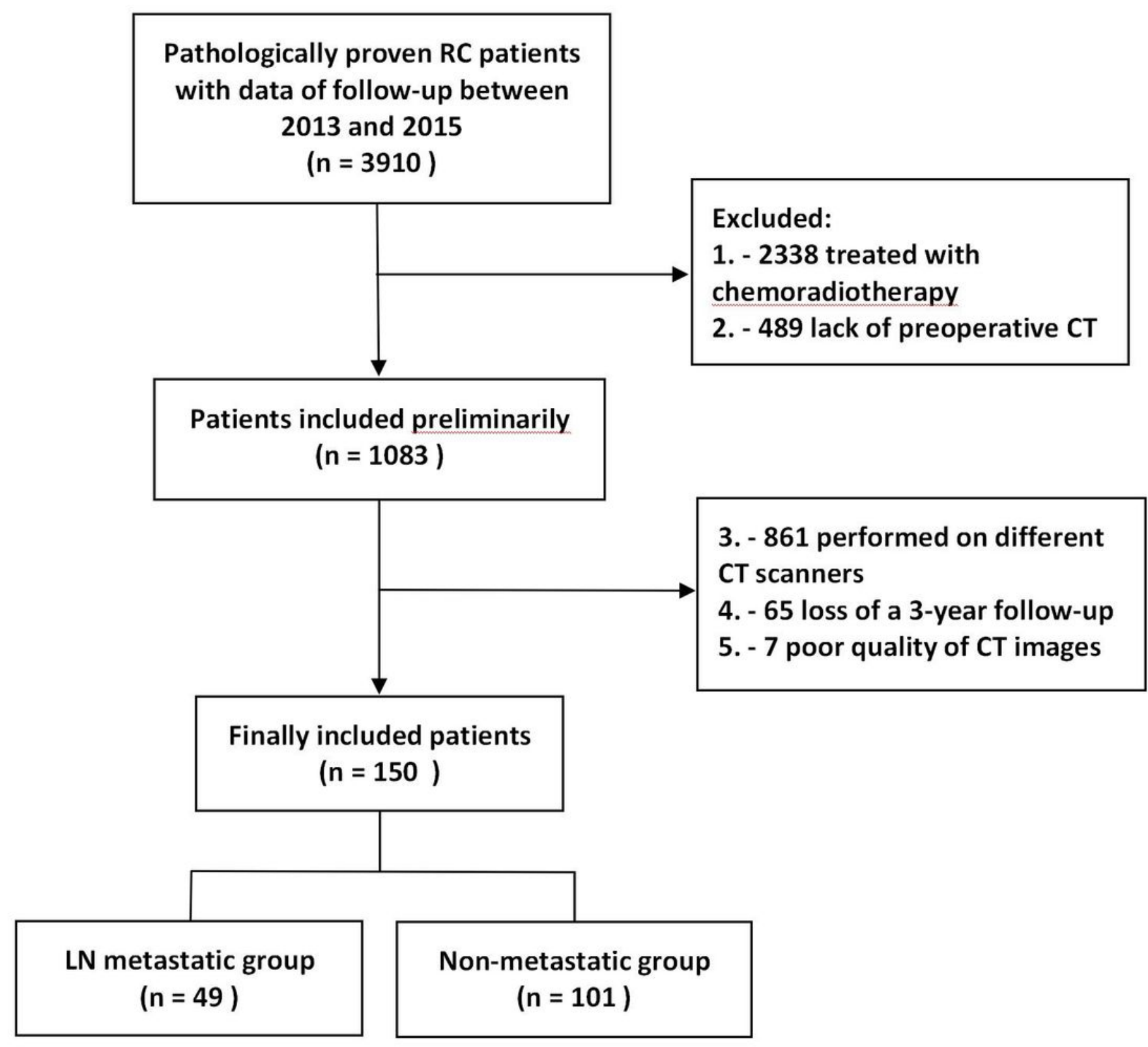

Figure 1

The flow diagram for patient enrollment. 

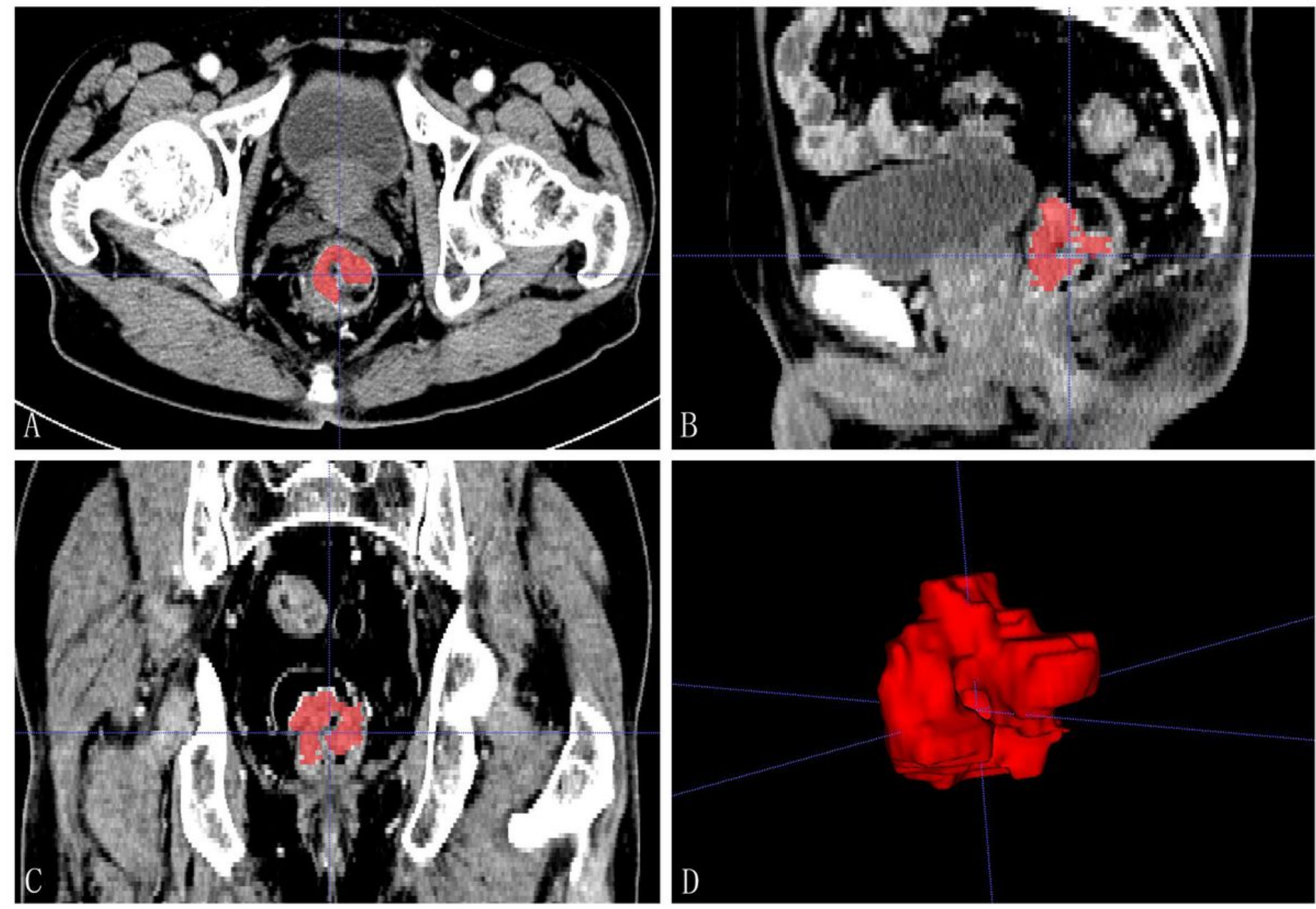

Figure 2

Regions of interest (ROIs) within a tumor. A-C: Manual segmentation of the entire lesion in the axial, sagittal and coronal enhanced CT. Red lines reflected the regions of interest used for radiomic feature. D: Three-dimensional volumetric reconstruction of the segmented lesion. 


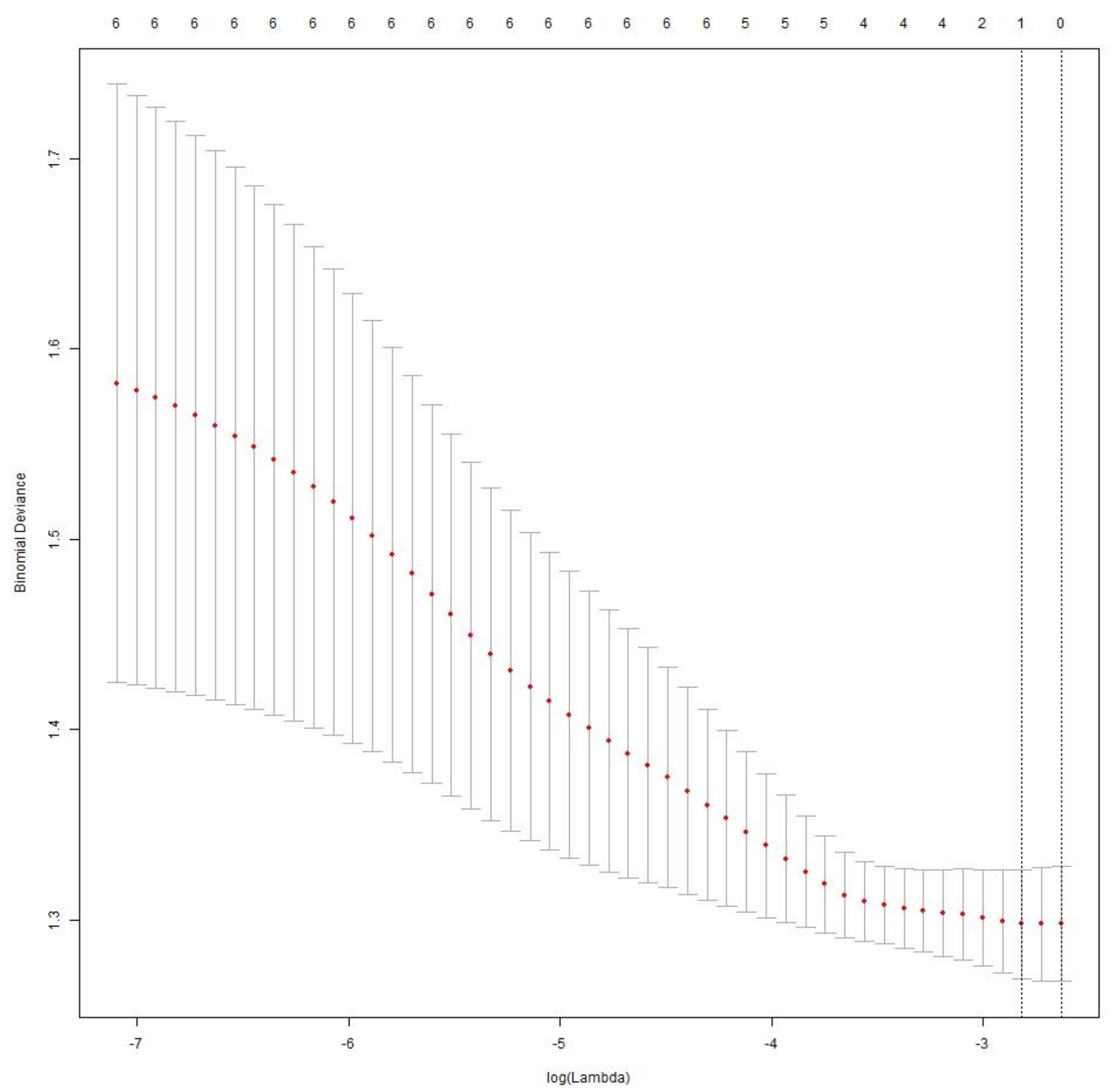

Figure 3

Radiomic feature selection by LASSO regression model. Vertical dotted lines were drawn to the optimum value (One feature was selected) using minimum criteria and 1 standard error criteria (1-SE criteria). 


\section{Nomogram}

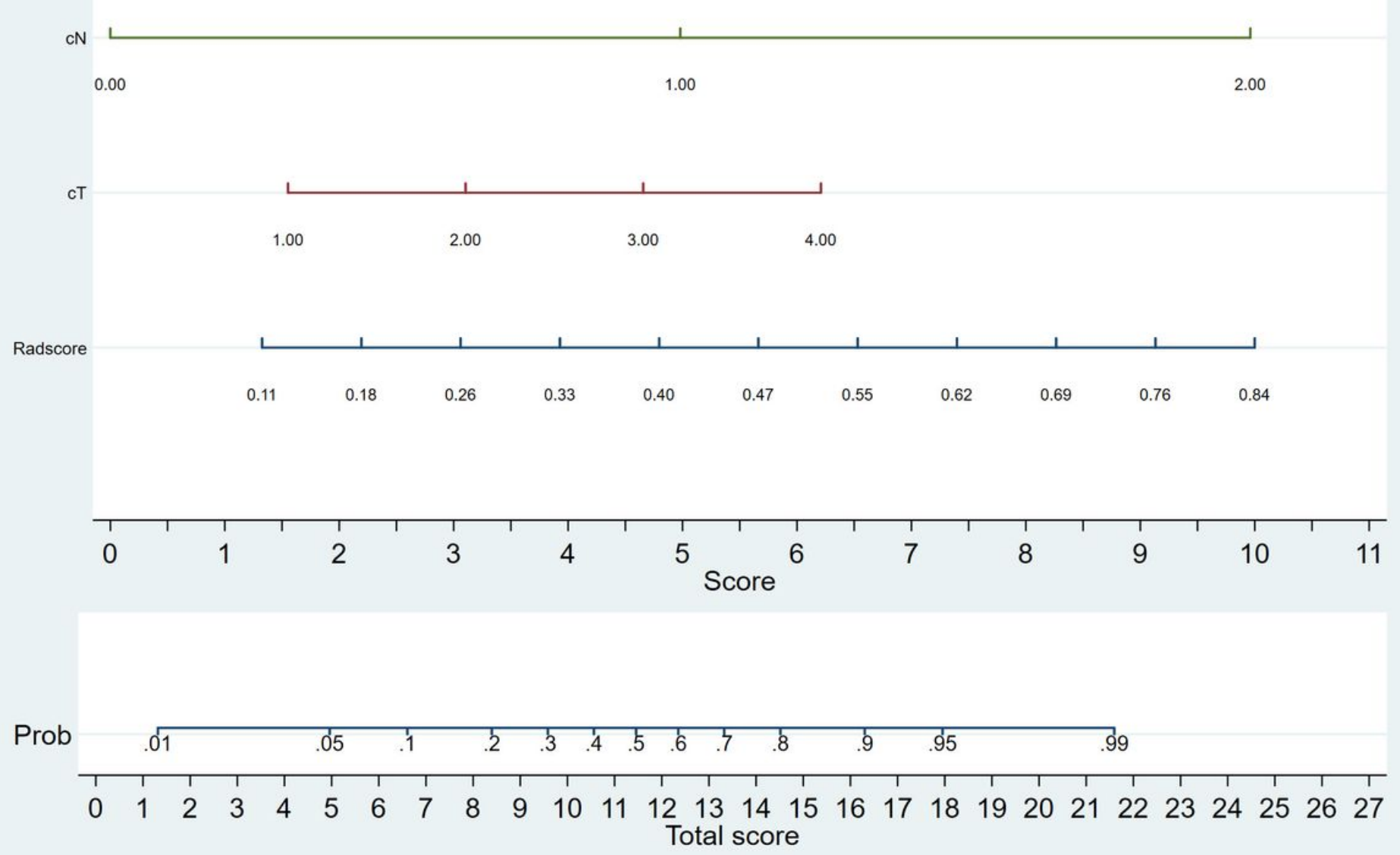

\section{Figure 4}

Nomogram for the combined model. The nomogram was established in the training cohort, with the incorporation of preoperative cT stage, $\mathrm{cN}$ stage and the Rad-score. 


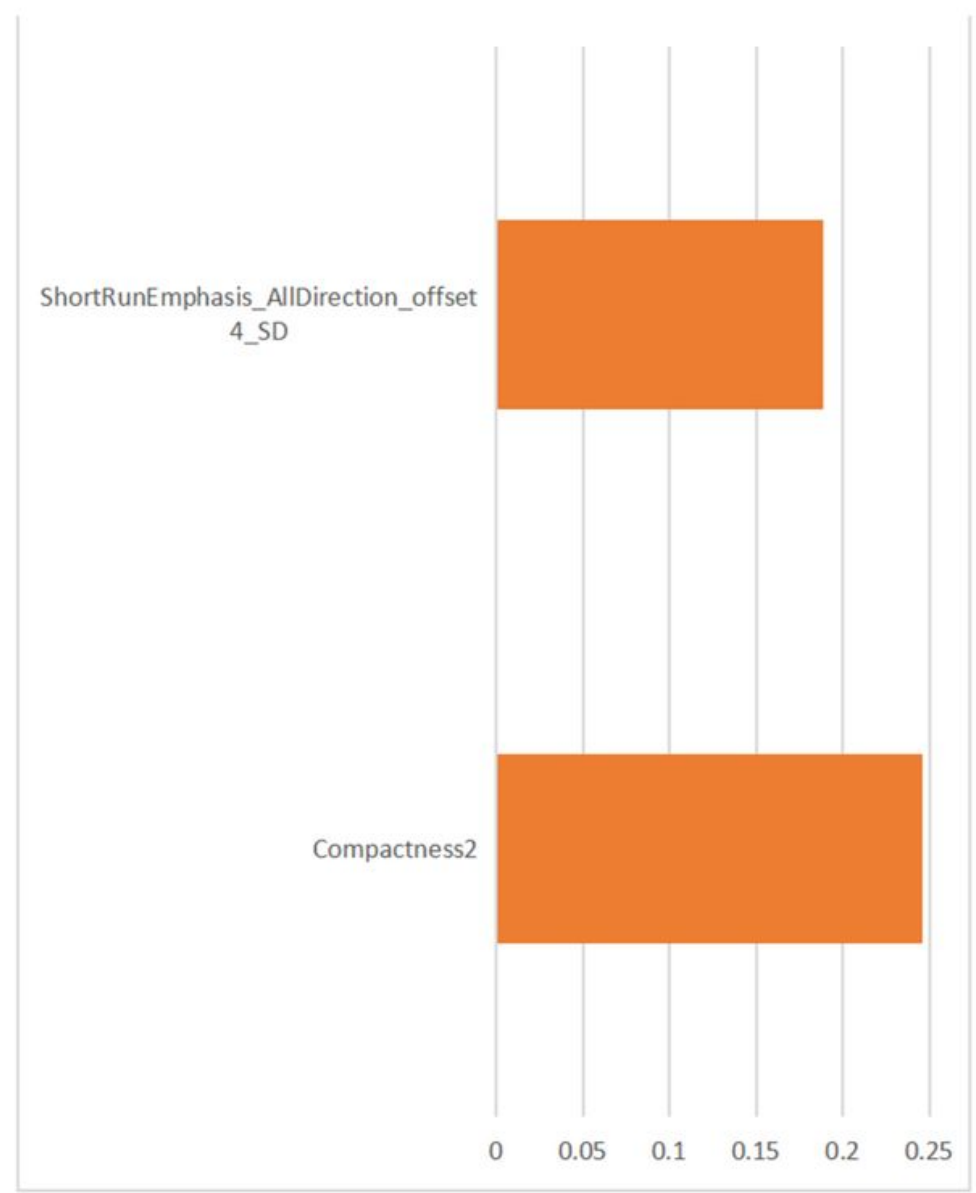

Radscore

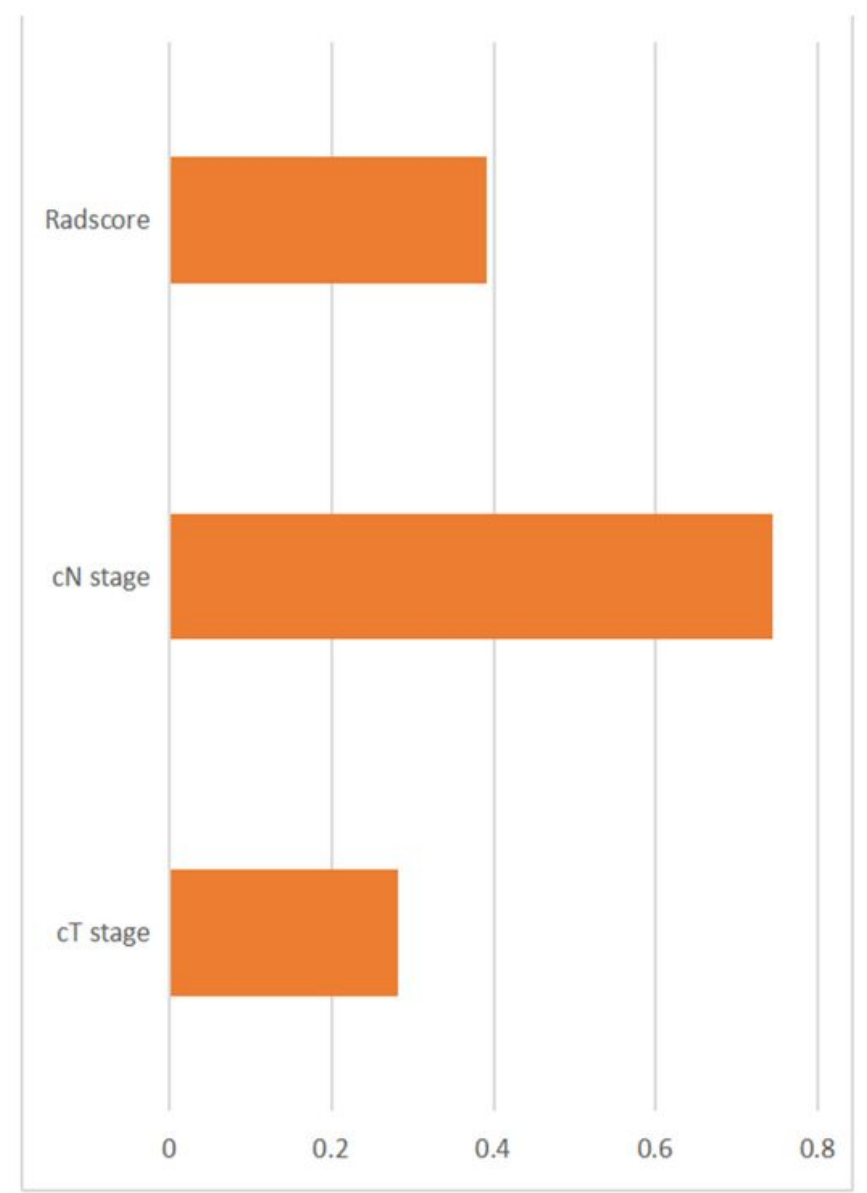

combined model

\section{Figure 5}

Contribution of each factors to Rad-score and the combined clinical-radiomic model. 


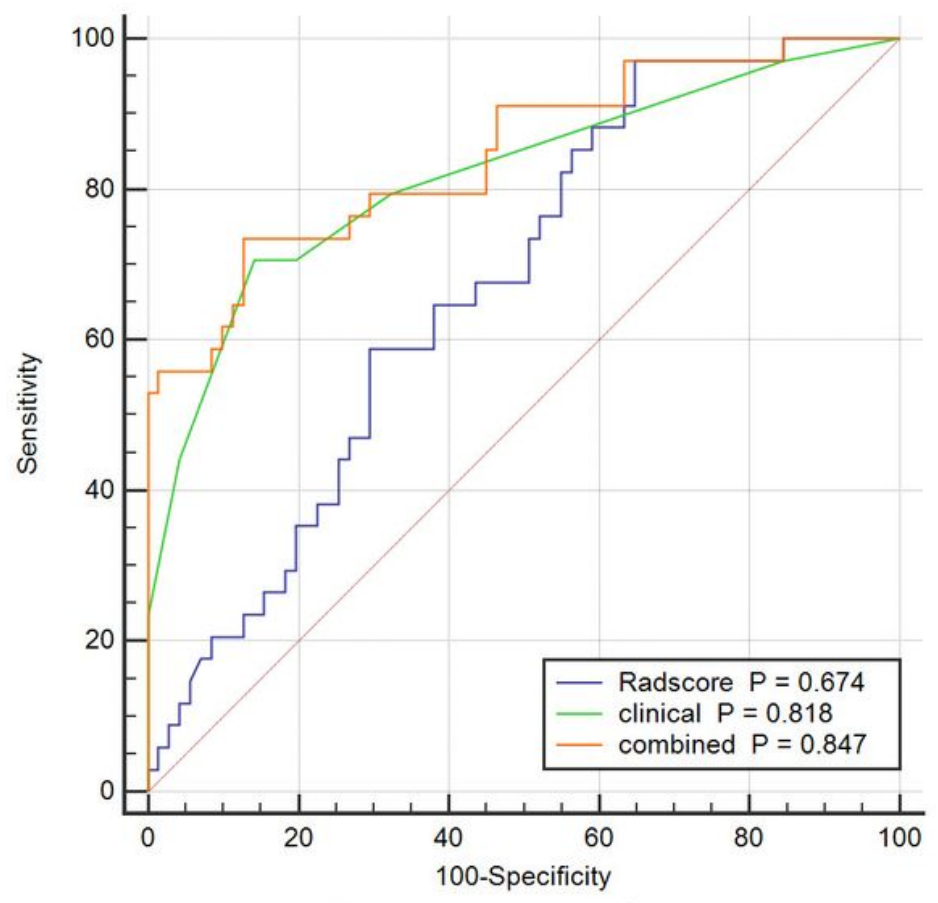

Training cohort

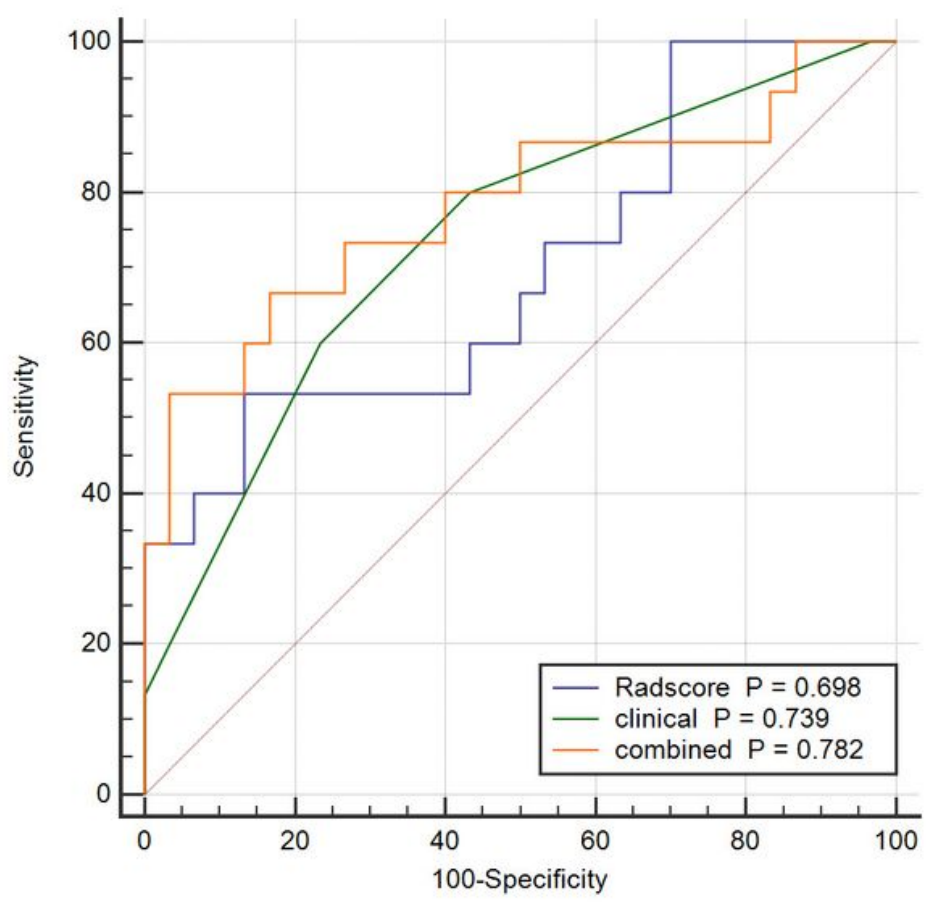

validation cohort

Figure 6

ROC curves of the combined model, clinical model (only preoperative cT stage and $\mathrm{cN}$ stage) and Radscore in the training and validation cohort.
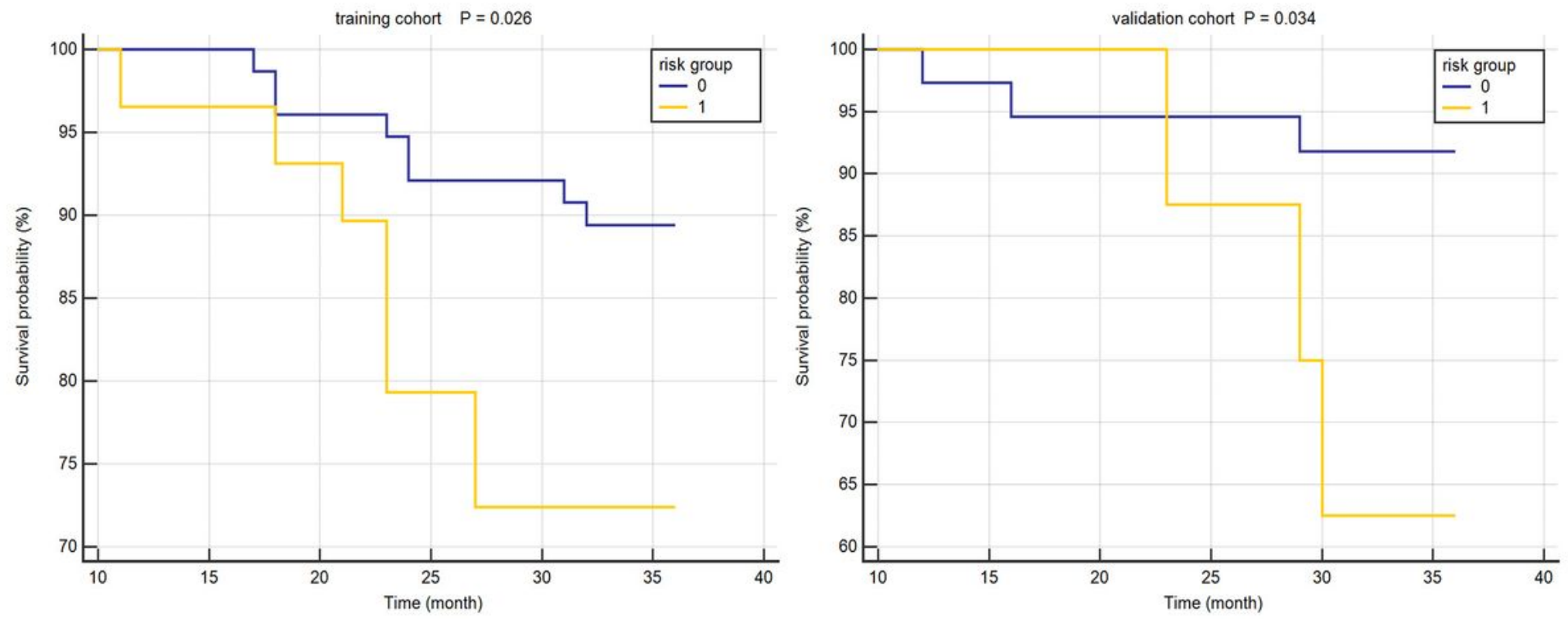

Figure 7

K-M curves for OS for different risk groups (stratified by the combined model) in both training and validation cohort. 